\title{
Security Parallel Migration of the Federal Cloud Markov Chain Multi Virtual Machine
}

\author{
Wu Lanhua ${ }^{1}$ \\ ${ }^{1}$ Department of Computer Science, Sichuan College of Applied Sciences, \\ Xichang City Sichuan Province, ZipCode615000, China \\ leacly@qq.com
}

\begin{abstract}
In order to improve the customer service quality during the virtual machine (VM) migration process in networks, a multi-VM parallel migration strategy based on Markov chain is proposed in this paper. Specifically, the simulation experiment is carried out to provide the data regarding the influence of the algorithm parameters on blocking probability, network migration time and downtime. According to the simulation result, under the condition of the same algorithm parameters, the multi-VM parallel migration process has low parallel downtime, and this index can significantly influence user experience improvement, thus indicating the advantage of the parallel strategy for improving user experience. Meanwhile, under the condition of the same parameters, the network blocking probability of the multi-VM parallel migration process is obviously lower than that of the sequence migration process, thus indicating that the parallel process has higher network utilization. Moreover, the network parameters can be properly selected according to actual needs.
\end{abstract}

Keywords: Cloud computing; Migration; Markov chain; Parallel computation; Virtual machine

\section{Introduction}

In recent years, the application software based on cloud computing mode has been widely researched and used in entertainment and business fields, and especially due to the realization of data center (DC) technology, "cloud" has been a ubiquitous computing tool. However, in order to respond to the exponentially increased cloud service users, especially to the significant increase of the mobile cloud users, many more advanced network infrastructures and technologies are deployed inside and outside DCs for information exchange. In fact, as long as there are available storage hardware conditions, any application program can be flexibly deployed in a server of any DC. Therefore, VM technology becomes the key technology for cloud computing service, wherein VM migration technology refers to the high-speed copying and transmission of the memory status of the original host computer to the target host computer. In practical application, such factors as network resources and memory dirty pages can influence VM migration performance; especially in the entertainment field, users much more concern the network service quality, thus proposing new requirements for VM migration technology [3]. How to reduce network migration time, network data transmission volume and other relevant indexes is excessively considered during the process of solving VM migration problems. Actually, under the background of the gradually increased network bandwidth, the influence of the network migration time is gradually reduced, but VM migration downtime index becomes more and more important and this index is closely related to user experience. Therefore, the mathematical analysis model for multi-VM parallel migration based on Markov chain is constructed in this paper from the angle of reducing 
the network downtime in order to improve the network computation utilization and reduce the network blocking rate and downtime, thus to improve user experience.

\section{Migration Model}

Multi-VM migration model is usually based on the following hypotheses:

(1) When $M_{z}$ VMs execute the migration process, for each migration request $z$, memory resource $V_{z}$ allocated thereby is consistent with other memory resources;

(2) The application programs running in VMs have the constant memory dirty page rate $D$;

(3) All VMs have the same fixed memory page size P;

(4) During the migration process, bit rate $R_{j}^{(z)}$ for requesting to concentrate the ${ }^{j \text { th }} V M$ migration is consistent with other bit rates, and this value is equal to the network channel capacity, for example: ${ }^{(z)} \leq b, \forall j=1, \cdots, M_{z}$.

$T_{i, j}^{(z)}$ is set as the time needed for the ${ }^{i}$ th iteration in the promotion stage for finishing $V M_{j}$ migration for each request $z$, and the total time computation formula for ${ }^{V M_{j}}$ migration is as follows[11]:

$$
\left\{\begin{array}{l}
T_{\text {mig, }, j}^{(z)}=\sum_{i=0}^{n_{j}^{(z)}} T_{i, j}^{(z)}=\sum_{i=0}^{n_{j}^{(z)}} \frac{(P D)^{i}}{\left(R_{j}^{(z)}\right)^{i+1}}=\frac{V_{z}}{R_{j}^{(z)}} \frac{1-\left(\gamma_{j}^{(z)}\right)^{n_{j}^{(z)}+1}}{1-\gamma_{j}^{(z)}} \\
n_{j}^{(z)}=\min \left\{\left[\log _{\gamma_{j}^{(z)}} \frac{V_{t h}}{V_{z}}\right], n_{\max }\right\}
\end{array}\right.
$$

Where $V_{\text {th }}$ is the dirty memory threshold value, ${ }^{n_{\max }}$ is the maximum iteration number for triggering the stop-copy stage, $\gamma_{j}^{(z)}=(P D) / R_{j}^{(z)}$ must be less than 1 , because the precopying migration algorithm can be continued only when the average dirty page rate of the memorizer is less than the transmission rate. The total migration time and the downtime of VMs set are strictly determined by the number of VMs in simultaneous migration. The two basic conditions are considered: (1) sequence migration: only one VM migration is executed at one time; (2) parallel migration: ${ }^{M} z$ VMs are simultaneously transferred. One useful parameter of the system is the specific value of the memory dirty page rate and the maximum transmission rate. When $M Z \mathrm{VMs}$ simultaneously execute the migration process, the full-rate transmission is executed, for example: $R_{j}^{(z)}=b, \forall j$. In such case, ${ }_{j}^{(z)}=\gamma, \forall j$.The sequence migration time of the whole VMs set $z$ is calculated as follows:

$T_{s-m i g}^{(z)}=\sum_{j=1}^{M_{z}} T_{m i g, j}^{(z)}=M_{z} \frac{V_{z}}{b} \frac{1-\gamma^{n_{s}^{(z)}+1}}{1-\gamma}$ 
Where $^{n_{s}^{(z)}}=\min \left\{\log _{\gamma} V_{t h} / V_{z}, n_{\max }\right\}$; as shown in Figure 2, the downtime of the whole VMs set is calculated since the termination of the first VM at the source host computer (namely since the last iteration of the first VM) ; afterwards, the last VM is restored at the target host computer. If $T_{\text {res }}$ is set as the fixed time needed for restoring a VM at the target host computer, then the downtime of the sequence migration can be calculated as follows:

$T_{s-d o w n}^{(z)}=\frac{V_{z}}{b} \gamma^{n_{s}^{(z)}}+\left(M_{z}-1\right) \frac{V_{z}}{b} \frac{1-\gamma^{n_{s}^{(z)}+1}}{1-\gamma}+T_{r e s}$

When ${ }^{M}$ VMs simultaneously execute the migration process, the migration rate of each VM depends on channel capacity ${ }^{b}$ needed for sharing continuous connection. Under the assumption that the channel capacity is uniformly decomposed, all VMs have the same memory configuration, start and end the iterations at the same moment, $M Z \mathrm{VMs}$ can be always found to have synchronous migration, the migration rate of each VM is $R_{j}^{(z)}=b / M_{z}, \forall j$. In such case, $\gamma_{j}^{(z)}=M_{z} \gamma, \forall j$. The parallel migration time of the whole VMs set is equal to any independent VM migration time:

$T_{p-m i g}^{(z)}=T_{m i g, j}^{(z)}=M_{z} \frac{V_{z}}{b} \frac{1-\left(M_{z} \gamma\right)^{n_{s}^{(z)}+1}}{1-M_{z} \gamma}$

$$
\text { Where } n_{p}^{(z)}=\min \left\{\left[\log _{M_{z} \gamma} V_{t h} / V_{z}\right], n_{\max }\right\}
$$

The parallel downtime of the whole VMs set is formally corresponding to the last iteration (the stop and copy stages) of any single VM as follows:

$T_{p-\text { down }}^{(z)}=M_{z} \frac{V_{z}}{b}\left(M_{z} \gamma\right)^{n_{s}^{(z)}}+T_{\text {res }}$

Where $T_{p-m i g}^{(z)} \geq T_{s-m i g}^{(z)}$ and $T_{p-\text { down }}^{(z)} \leq T_{s-d o w n}^{(z)}$. This means that parallel migration can provide the terminal service with higher quality due to less downtime, and the sequence migration has lower communication and computation resource overheads due to less total transmission time. 


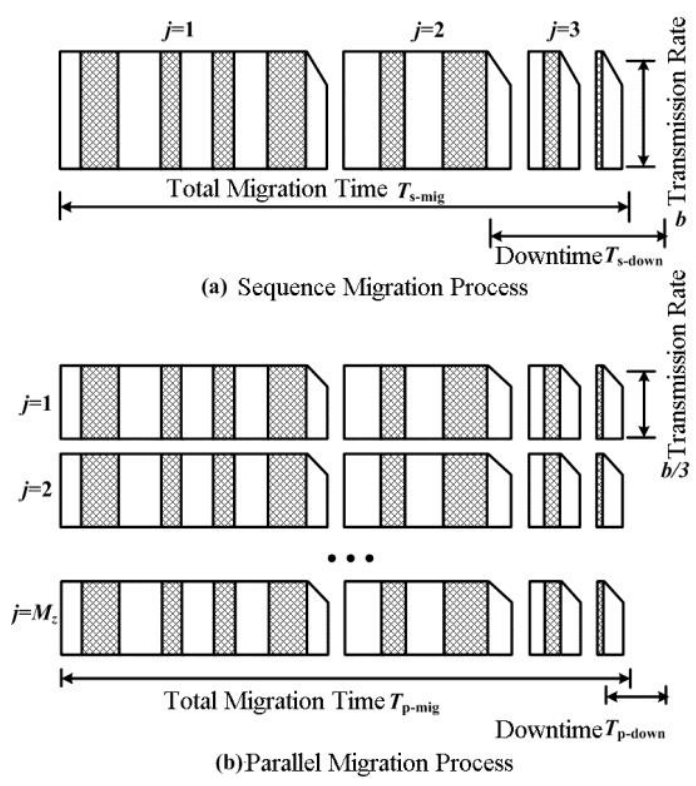

Figure 1. Sequence Migration and Parallel Migration Process

\section{Model Analysis}

Except migration time and downtime, other parameters are defined as the total VM migration cost for quantitative evaluation. One of the cost parameters is VM performance loss, and in consideration of the overhead for monitoring the memory write access operations, this parameter can significantly delay the execution process in VM and reduce the throughput. Another important cost parameter is the energy overhead, namely the additional energy consumed by the computation and communication resources during VM migration process. Specifically, the following two parameters are measured to quantify the migration cost of VMs set.

(1) The data transmission volume in the network channel during the migration process represents the communication resource overhead;

(2) The volume of the data, which the application program running in a VM cannot adopt to exchange with external users during the downtime, represents the performance degradation index of VM process.

The communication resource overheads of the sequence migration and the parallel migration are respectively $T_{s-m i g}^{(z)} b$ and $T_{p-m i g}^{(z)} b$; the later index can be obtained through setting the throughput of the application program equal to the transmission rate, for example, $T_{s-\text { down }}^{(z)} b$ and $T_{p-d o w n}^{(z)} b$. In order to feasibly compare the two network costs, ${ }^{\gamma}$ is set approximately to the upper bound thereof to find the upper limit of each index:

$$
\begin{gathered}
\lim _{\gamma \rightarrow 1} T_{s-m i g}^{(z)} b=M_{z} V_{z} \lim _{\gamma \rightarrow 1} \frac{1-\gamma^{n_{s}^{(z)}+1}}{1-\gamma}=M_{z} V_{z}\left(n_{\max }+1\right) \\
\lim _{\gamma \rightarrow 1 / M_{z}} T_{p-m i g}^{(z)} b=M_{z} V_{z} \lim _{\gamma \rightarrow 1 / M_{z}} \frac{1-\left(M_{z} \gamma\right)^{n_{p}^{(z)}+1}}{1-M_{z} \gamma} \\
=M_{z} V_{z}\left(n_{\max }+1\right)
\end{gathered}
$$


$\lim _{\gamma \rightarrow 1} T_{s-\text { down }}^{(z)} b=V_{z}\left(1+\left(M_{z}-1\right)\left(n_{\max }+1\right)\right)+T_{\text {res }} b$

$\lim _{\gamma \rightarrow 1 / M_{z}} T_{p-d o w n}^{(z)} b=M_{z} V_{z}+T_{r e s} b$

Therefore, VM migration costs for the sequence migration and the parallel migration respectively executed for each request $\mathrm{Z}$ can be expressed as follows in the forms of the network overhead and the throughput of the application program:

$c_{s-n e t}^{(z)}=\left(1-\gamma^{n_{s}^{(z)}+1}\right) /\left[\left(n_{\max }+1\right)(1-\gamma)\right]$

$c_{p-n e t}^{(z)}=\left(1-\left(M_{z} \gamma\right)^{n_{p}^{(z)}+1}\right) /\left[\left(n_{\max }+1\right)\left(1-M_{z} \gamma\right)\right]$

$c_{s-a p p}^{(z)}=\frac{V_{z}\left(\gamma^{n_{s}^{(z)}}+\left(M_{z}-1\right) \frac{1-\gamma^{n_{s}^{(z)}+1}}{1-\gamma}\right)+T_{\text {res }} b}{V_{z}\left(1+\left(M_{z}-1\right)\left(n_{\max }+1\right)\right)+T_{r e s} b}$

$c_{p-a p p}^{(z)}=\frac{M_{z} V_{z}\left(M_{z} \gamma\right)^{n_{p}^{(z)}}+T_{r e s} b}{M_{z} V_{z}+T_{r e s} b}$

Through weighting, VM migration costs for the sequence migration and the parallel migration respectively executed for each request $\mathrm{Z}$ can be formally obtained as follows:

$\left\{\begin{array}{l}c_{s}^{(z)}=\alpha c_{s-n e t}^{(z)}+(1-\alpha) c_{s-a p p}^{(z)} \\ c_{p}^{(z)}=\alpha c_{p-n e t}^{(z)}+(1-\alpha) c_{p-a p p}^{(z)}\end{array}\right.$

Where the weight parameter is set as $0 \leq \alpha \leq 1$.

\section{Experiment and Analysis}

For Markov parallel execution model based on the federal cloud network, the network performance indexes thereof are tested under different experimental parameters in this chapter. VM memory size is assumed as $V_{z}$ and follows the bimodal distribution. Namely, the following conditions can be met [12]:

$\left\{\begin{array}{l}V_{z}=V_{0}, \quad \text { s.t.probability }=q \\ V_{z}=n V_{0}, \text { s.t.probability }=1-q\end{array}\right.$

VM memory size requirement can be obtained according to the specific operation condition of the application program. Meanwhile, VM quantity of each migration cluster is assumed to follow the uniform distribution in the interval of $1 \sim M$. Therefore, the average multi-VM migration time under continuous condition can be calculated as follows [13]:

$E\left[T_{s-m i g}^{(z)}\right]=\frac{M+1}{2} \frac{V_{0}}{b} \frac{q f_{z}+u(1-q) f_{z}}{1-\gamma}$ 
Where $n_{s}=\min \left\{\left[\log _{\gamma} V_{t h} / x\right], n_{\max }\right\}$ and $f_{z}=1-\gamma^{n_{s} V_{0}+1}$. The experimental parameters are set as follows: VM quantity: $M=2$; small VM memory size: $V_{0}=1 \mathrm{~GB}$; large VM memory factor: $u=4$; small VM proportion: $q=0.75$; dirty page rate: $D=2500 \mathrm{pps}$; memory page size: $p=4 \mathrm{~KB}$; threshold value of residual dirty page memory size: $\mathrm{V}_{t h}=100 \mathrm{MB}$; maximum iteration times: ${ }^{n_{\max }}=8$; VM restoring time: $T_{\text {res }}=100 \mathrm{~ms}$;

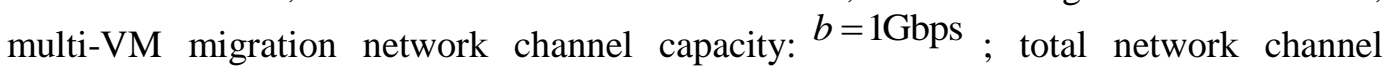
capacity: $B=4 \mathrm{Gbps}$; remote DCS quantity: $n=5$; resource set quantity of each request: $m=3$; quantity of the loads supported by each remote DC: $k=8$. Figure 2 shows the change of the migration request blocking rate along with the independent variable ---migration request arrival rate ${ }^{\lambda}$ (unit: req/min ) under different network channel capacities $B$. Meanwhile, the sequence migration strategy and the parallel migration strategy are respectively considered for comparison.

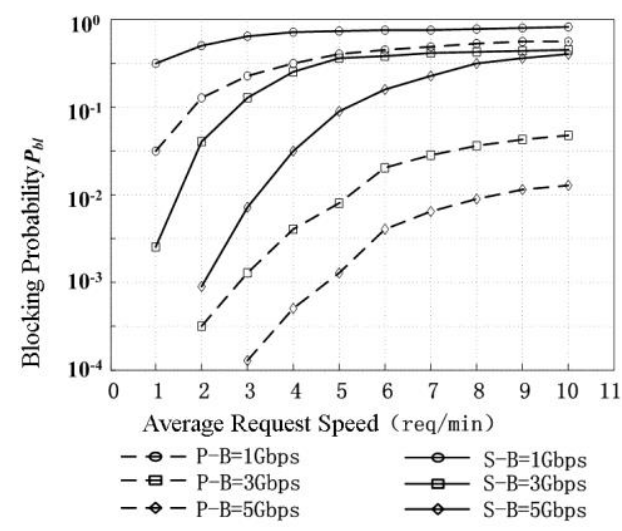

\section{Figure 2. Arrival Rate - Blocking Rate Function}

In Figure 2, $S-B=1 \mathrm{Gbps}$ represents the blocking probability of the sequence execution under total channel capacity $B=1 \mathrm{Gbps}$. According to the above figure, the migration request blocking probability is gradually increased along with arrival rate $\lambda$ and gradually becomes saturated. Obviously, under the model sequence and the parallel mode, the blocking probability is reduced along with the increase of channel capacity $B$, and this is consistent with the practical condition. Additionally, Figure 3 shows the change curve of the migration request blocking probability along with migration request arrival rate ${ }^{\lambda}$ under different remote DCs.

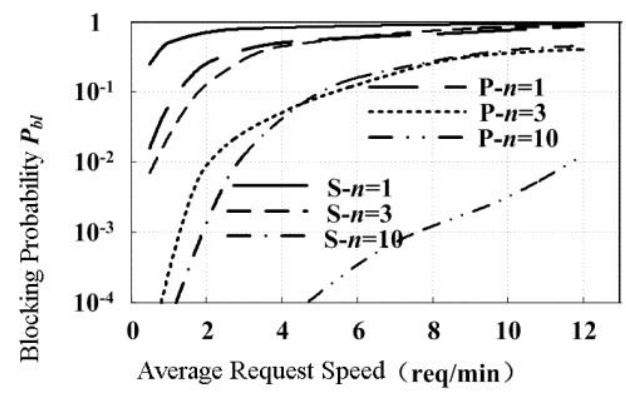

Figure 3. The Influence of the Number of Remote dcs on the Blocking Probability 
In Figure 3, $S-n=1$ represents the blocking probability of the sequence migration when there is only one remote DC. According to Figure 4, under the sequence model and the parallel mode, the blocking probability is reduced along with the increase of remote DCs quantity. Meanwhile, Figure 4 also shows the migration request blocking probabilities of the sequence migration and the parallel migration under different maximum VMs quantities for each request.

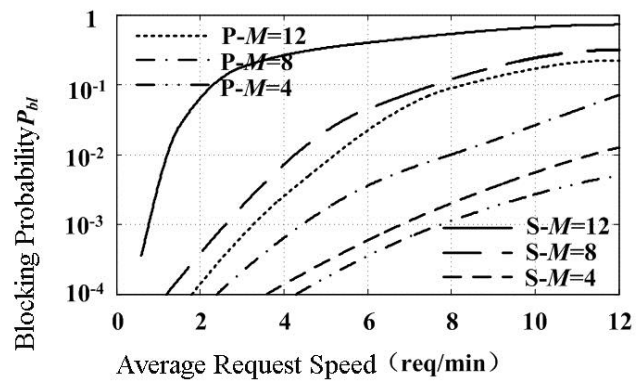

Figure 4. The Influence of the Upper Limit of the Virtual Machine Transfer on the Blocking Probability

According to the experiment results as shown in Figure 4, under the sequence migration strategy and the parallel migration strategy, the blocking probability is reduced along with the reduction of the upper limit of VM transfer quantity. In practical situation, the increase of the transfer quantity at each time can easily cause large impact to the network channel and accordingly increase the blocking probability. Meanwhile, Figure 5 shows the changes of the migration time and the downtime along with network channel capacity ${ }^{b}$. In this section, the sequence migration strategy and the parallel migration strategy are also respectively considered for comparison.

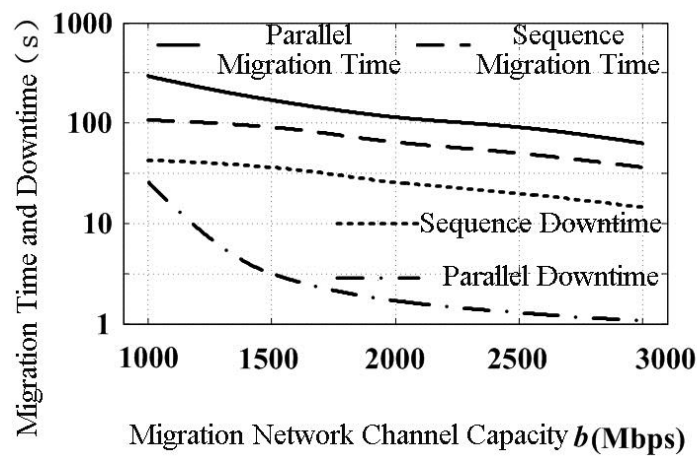

Figure 5. Migration Time and Downtime Changes

Figure 5 shows the changes of the migration time and the downtime along with network channel capacity $b$ respectively under sequence migration strategy and parallel migration strategy. Obviously, the migration time and the downtime are both reduced along with the increase of migration network channel capacity ${ }^{b}$, thus indicating that the migration performance can be improved through increasing the hardware bandwidth investment. Meanwhile, according to the comparison between the two strategies, under the same migration network channel capacity ${ }^{b}$, the parallel migration time is longer than the sequence migration time and the parallel downtime is less than the sequence downtime, thus indicating that the parallel strategy is favorable for improving the service quality during VM migration process. Additionally, Figure 6 shows the comparison curves of the network migration costs expressed by Formula (14) respectively under the sequence migration strategy and the parallel migration strategy. 


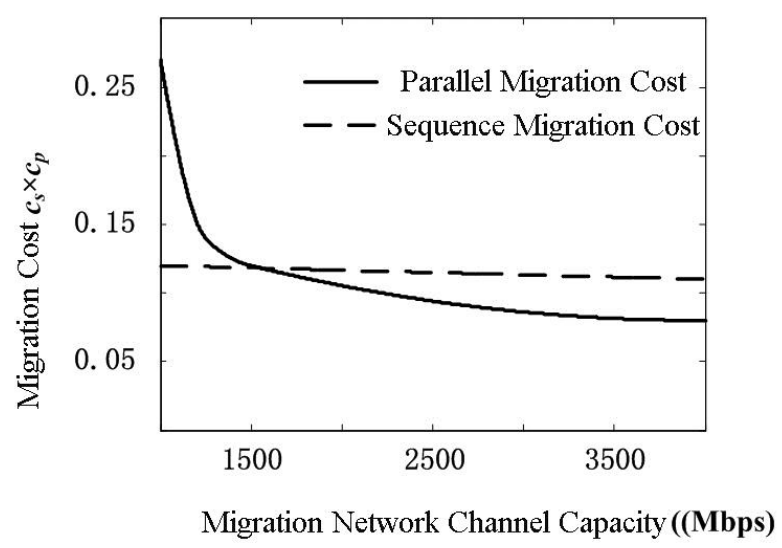

Figure 6. Migration Costs Comparison

According to the migration costs comparison curve shown in Figure 6, under small network migration channel capacity ( $b \leq 1500$ ), the parallel migration cost is obviously higher than the sequence migration cost, thus indicating that the parallel migration needs more network channel resources. Along with the increase of the channel capacity, the parallel migration cost is less than the sequence migration cost, thus presenting the parallelization advantage for big data processing.

\section{Conclusion}

In allusion to the influence of the migration time and the downtime on user experience during VM migration process, the parallel migration strategy of the feral cloud Markov chain multi-VM is proposed in this paper from the angle of improving the network service quality and reducing the network operation cost. Firstly, the traditional sequence migration process is researched in order to research and construct the multi-VM parallel migration process and meanwhile provide the computation model for calculating the migration time and the downtime. Then, the multi-VM parallel migration is conceived into a Poisson distribution process and the mathematical analysis model for the multi-VM parallel migration is constructed on the basis of Markov chain. Finally, relevant experiment is carried out to describe the influence of the model parameters on the multiVM migration process and compare the sequence migration process with the parallel migration process in the aspects of migration time, downtime and network cost so as to verify the advantages of the parallel migration process for processing the big data migration.

\section{References}

[1] J. Hu and Z. Gao, "Modules identification in gene positive networks of hepatocellular carcinoma using Pearson agglomerative method and Pearson cohesion coupling modularity[J]", Journal of Applied Mathematics, 2012, (2012).

[2] Yang J., He S. and Lin Y., "Multimedia cloud transmission and storage system based on internet of things[J]", Multimedia Tools and Applications, (2015).

[3] Guo C., Liu X. and Jin M., "The research on optimization of auto supply chain network robust model under macroeconomic fluctuations[J]", Chaos, Solitons \& Fractals, (2015).

[4] Li X., Lv Z. and Hu J., "XEarth: A 3D GIS Platform for managing massive city information[C]", //Computational Intelligence and Virtual Environments for Measurement Systems and Applications (CIVEMSA), 2015 IEEE International Conference on. IEEE, (2015), pp. 1-6.

[5] Yang J., Chen B. and Zhou J., "A Low-Power and Portable Biomedical Device for Respiratory Monitoring with a Stable Power Source[J]”, Sensors, vol. 15, no. 8, (2015), pp. 19618-19632.

[6] G. Bao, L. Mi, Y. Geng and K. Pahlavan, "A computer vision based speed estimation technique for localiz ing the wireless capsule endoscope inside small intestine", 36th Annual International Conference of the IEEE Engineering in Medicine and Biology Society (EMBC), (2014) Aug. 
[7] Lv Z., Halawani A. and Feng S., "Multimodal hand and foot gesture interaction for handheld devices[J]", ACM Transactions on Multimedia Computing, Communications, and Applications (TOMM), vol. 11, no. 1s, (2014), pp. 10.

[8] G. Liu, Y. Geng and K. Pahlavan, "Effects of calibration RFID tags on performance of inertial navigation in indoor environment", 2015 International Conference on Computing, Networking and Communications (ICNC), (2015) Feb.

[9] Su T., Wang W. and Lv Z, "Rapid Delaunay triangulation for randomly distributed point cloud data using adaptive Hilbert curve[J]", Computers \& Graphics, vol. 54, (2016), pp. 65-74.

[10] Gu W., Lv Z. and Hao M., "Change detection method for remote sensing images based on an improved Markov random field[J]", Multimedia Tools and Applications, (2015), pp. 1-16.

[11] Lv Z., Tek A. and Da Silva F., "Game on, science-how video game technology may help biologists tackle visualization challenges[J]", PloS one, vol. 8, no. 3, (2013), 57990.

[12] Chen Z., Huang W. and Lv Z., "Towards a face recognition method based on uncorrelated discriminant sparse preserving projection[J]", Multimedia Tools and Applications, (2015), pp. 1-15.

[13] Jiang D., Ying X. and Han Y., "Collaborative multi-hop routing in cognitive wireless networks[J]", Wireless Personal Communications, (2015), pp. 1-23.

[14] Lv Z., Tek A. and Da Silva F., "Game on, science-how video game technology may help biologists tackle visualization challenges[J]", PloS one, vol. 8, no. 3, (2013), 57990.

[15] Jiang D., Xu Z. and Lv Z., "A multicast delivery approach with minimum energy consumption for wireless multi-hop networks[J]", Telecommunication Systems, (2015), pp. 1-12.

[16] Lv Z., Yin T. and Han Y., "WebVR—— web virtual reality engine based on P2P network[J]", Journal of Networks, vol. 6, no. 7, (2011), pp. 990-998.

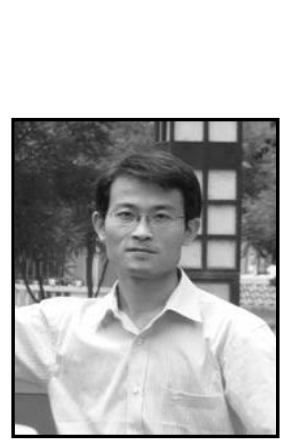

\section{Authors}

Wu Lanhua, He graduated from Northwest Normal University with a bachelor's degree in mathematics and information science. And he is a graduate of the education School in Shaanxi Normal University. His research interests include application and development of computer education technology, the application of cloud computing technology and the application of virtualization technology .He was a lecturer at department of computer science and the director at computer teaching center in Shaanxi fashion engineering university, and he was a lecturer of Computer teaching and research section at Chengdu institute Sichuan international studies university. Currently he is a lecturer at Department of Computer Science, Sichuan College of Applied Sciences. 
International Journal of Security and Its Applications

Vol. 10, No. 8 (2016) 